\title{
Sequencing of E. coli strain UTI89 on multiple sequencing platforms
}

\author{
Shannon N. Fenlon' ${ }^{1}$ Yuemin Celina Chee ${ }^{2}$, Jacqueline Lai Yuen Chee ${ }^{1}$, Yeen Hui Choy ${ }^{1}$, Alexis Jiaying Khng ${ }^{1}$, \\ Lu Ting Liow ${ }^{2}$, Kurosh S. Mehershahi ${ }^{2}$, Xiaoan Ruan', Stephen W. Turner ${ }^{3}$, Fei Yao ${ }^{1}$ and Swaine L. Chen ${ }^{1,2^{*}}$ (D)
}

\begin{abstract}
Objectives: The availability of matched sequencing data for the same sample across different sequencing platforms is a necessity for validation and effective comparison of sequencing platforms. A commonly sequenced sample is the lab-adapted MG1655 strain of Escherichia coli; however, this strain is not fully representative of more complex and dynamic genomes of pathogenic E. coli strains.

Data description: We present six new sequencing data sets for another E. coli strain, UTI89, which is an extraintestinal pathogenic strain isolated from a patient suffering from a urinary tract infection. We now provide matched whole genome sequencing data generated using the PacBio RSII, Oxford Nanopore MinION R9.4, Ion Torrent, ABI SOLiD, and Illumina NextSeq sequencers. Together with other publically available datasets, UTI89 has a nearly complete suite of data generated on most second- and third-generation sequencers. These data can be used as an additional validation set for new sequencing technologies and analytical methods. More than being another E. coli strain, however, UTI89 is pathogenic, with a 10\% larger genome, additional pathogenicity islands, and a large plasmid, features that are common among other naturally occurring and disease-causing E. coli isolates. These data therefore provide a more medically relevant test set for development of algorithms.
\end{abstract}

Keywords: Escherichia coli, UPEC, Urinary Tract Infection (UTI), Ion Torrent, SOLiD, Illumina, Oxford Nanopore, MinION, PacBio, Roche454

\section{Objective}

Control sequencing data across different sequencing platforms is extremely important for validation and effective comparison of sequencing platforms. A commonly sequenced sample that has been extensively used for these purposes is the MG1655 strain of E. coli [1]. However, the MG1655 genome is smaller and less complex than those of some pathogenic E. coli strains [2, 3]. As part of control experiments, we have sequenced UTI89, a uropathogenic E. coli (UPEC) strain originally isolated from a patient suffering from an acute bladder

\footnotetext{
*Correspondence: slchen@gis.a-star.edu.sg

1 Genome Institute of Singapore, 60 Biopolis Street, Genome, \#02-01,

Singapore 138672, Singapore

Full list of author information is available at the end of the article
}

infection [4], using several different sequencing technologies, including ABI SOLiD, Ion Torrent, PacBio, Oxford Nanopore, and Illumina. Our new data supplements previously published sequencing data generated using the Roche 454 [4], Illumina HiSeq [5], and the original Oxford Nanopore Technologies MinION [6]. With the inclusion of these new data sets, E. coli strain UTI89 now has a nearly complete set of raw sequence data generated using most second- and third-generation sequencers. For some of the technologies we have multiple data sets, such as for PacBio, which spans the first iteration of the RSII sequencing chemistry $(\mathrm{XL} / \mathrm{C} 2)$ in 2012 up to the P6-C4 chemistry (which was current in 2018), which led to a more than fivefold increase in mean read length.

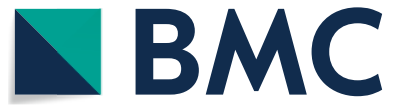

(c) The Author(s) 2020. This article is licensed under a Creative Commons Attribution 4.0 International License, which permits use, sharing, adaptation, distribution and reproduction in any medium or format, as long as you give appropriate credit to the original author(s) and the source, provide a link to the Creative Commons licence, and indicate if changes were made. The images or other third party material in this article are included in the article's Creative Commons licence, unless indicated otherwise in a credit line to the material. If material is not included in the article's Creative Commons licence and your intended use is not permitted by statutory regulation or exceeds the permitted use, you will need to obtain permission directly from the copyright holder. To view a copy of this licence, visit http://creativecommons.org/licenses/by/4.0/. The Creative Commons Public Domain Dedication waiver (http://creativecommons.org/publicdomain/zero/1.0/) applies to the data made available in this article, unless otherwise stated in a credit line to the data. 


\section{Data description}

The new data sets are summarized in Table 1. Details of library preparation and sequencing methods for the new datasets are presented below.

\section{SOLiD}

\section{Library preparation}

Genomic DNA was extracted from UTI89 grown overnight in Lysogeny Broth (LB) and used to generate Long Mate Pair (LMP) libraries. LMP libraries were generated using an insert size of $3-4 \mathrm{~kb}$ according to the manufacturer's instructions to produce a 375 bp library.

\section{Sequencing}

A $2 \times 35 b p$ LMP sequencing run was performed on two spots of an 8 spot slide using the Applied Biosystems SOLiD3 platform [7-9].

\section{Ion Torrent}

\section{Library preparation}

Genomic DNA was extracted from UTI89 harbouring the pBAD33 plasmid [10] grown overnight in LB. Sequencing libraries were then generated using the Ion Xpress ${ }^{\mathrm{TM}}$ Plus gDNA library preparation protocol according to the manufacturer's instructions.

\section{Sequencing}

A $200 \mathrm{bp}$ sequencing run was performed on the personal genome machine (PGM) system using the Ion $\mathrm{PGM}^{\mathrm{TM}} 200$ Sequencing Kit with a 316 chip $[11,12]$.

\section{PacBio, RSII, XL/C2 Chemistry \\ Library preparation}

Genomic DNA was extracted from SLC-66 (UTI89 with a kanamycin cassette integrated into the phage HK022 integration site) grown overnight in LB. Large insert (15 Kb) native SMRTbell sequencing libraries were generated according to the manufacturer's protocols.

\section{Sequencing}

Sequencing was performed on 6 SMRT Cells using XL/ C2 Sequencing chemistry [13-15].

\section{Illumina}

\section{Library preparation}

Genomic DNA was extracted from UTI89 grown overnight in LB. Sequencing libraries were built using the
Illumina TruSeq Nano DNA LT kit according to the manufacturer's instructions, with shearing to $350 \mathrm{bp}$.

\section{Sequencing}

A 2x150bp sequencing run was performed using the Illumina NextSeq 500 and a NextSeq Mid Output flow cell and reagents $[16,17]$.

\section{Oxford Nanopore, MinION Mk1B Device, R9.4, 1D Ligation} sequencing

\section{Library preparation}

Genomic DNA was extracted from UTI89 grown overnight in LB. $1 \mu \mathrm{g}$ of unsheared DNA was used to prepare sequencing libraries using the Ligation sequencing kit $1 \mathrm{D}$ R9 version (SQK-LSK108) according to the manufacturer's instructions.

\section{Sequencing}

The prepared sequencing library was loaded onto a FLOMIN106 R9.4 with Spot-ON and a $24 \mathrm{~h}$ sequencing run was performed. Base calling was subsequently performed using Oxford Nanopore's Albacore Sequencing Pipeline Software (version 1.2.1) [18, 19].

\section{PacBio, RSII, P6-C4 Chemistry \\ Library preparation}

Genomic DNA was extracted from UTI89 grown overnight in LB. Large insert $(20 \mathrm{~Kb})$ native SMRTbell sequencing libraries were generated according to the manufacturer's instructions.

\section{Sequencing}

Sequencing was performed on 2 SMRT Cells using P6-C4 Sequencing chemistry [20-23].

\section{Previously published data sets}

There are three previously published data sets generated using other sequencing platforms or sequencer versions: Roche 454 [4, 24-30], Illumina HiSeq 2000 [5, 31-34], and the original Oxford Nanopore MinION with an R7 flow cell $[6,35,36]$. The data presented in this manuscript complements these published datasets (also included in Table 1).

\section{Limitations}

The following are limitations of these data:

1. The data was collected over a period of several years, and thus all experimental steps were performed by different persons.

2. Some strains contain plasmids or other markers (see details above). 
Table 1 Overview of data sets

\begin{tabular}{|c|c|c|c|}
\hline Label & Name of data file/data set & $\begin{array}{l}\text { File types } \\
\text { (file extension) }\end{array}$ & $\begin{array}{l}\text { Data repository and identifier (DOI } \\
\text { or accession number) }\end{array}$ \\
\hline Data set 1 & New UTI89 genomic data & XLSX (containing data on FASTQ files) & $\begin{array}{l}\text { Figshare (https://doi.org/10.6084/ } \\
\text { m9.figshare.12195663) }\end{array}$ \\
\hline Data set 2 & $\begin{array}{l}\text { Previously published UTI89 } \\
\text { genomic data }\end{array}$ & XLSX (containing data on FASTQ files) & $\begin{array}{l}\text { Figshare (https://doi.org/10.6084/ } \\
\text { m9.figshare.12195675) }\end{array}$ \\
\hline Applied Biosystems SOliD 3 (new) & UTI89 - SoliD 3 LMP & FASTQ & $\begin{array}{l}\text { NCBI Sequence Read Archive (https:// } \\
\text { identifiers.org/ncbi/insdc.sra:SRX43 } \\
87579 \text { [7]; https://identifiers.org/ } \\
\text { ncbi/insdc.sra:SRR7517573 [8]; } \\
\text { https://identifiers.org/ncbi/insdc } \\
\text {.sra:SRR8247388 [9]) }\end{array}$ \\
\hline Ion Torrent PGM (new) & UTI89/pBAD33 - IonTorrent & FASTQ & $\begin{array}{l}\text { NCBI Sequence Read Archive (https:// } \\
\text { identifiers.org/ncbi/insdc.sra:SRX42 } \\
25380 \text { [11]; https://identifiers.org/ } \\
\text { ncbi/insdc.sra:SRR7352157 [12]) }\end{array}$ \\
\hline Pacific Biosciences RSII (XL/C2) (new) & SLC-66 - PacBio XL/C2 & FASTQ & $\begin{array}{l}\text { NCBI Sequence Read Archive (https:// } \\
\text { identifiers.org/ncbi/insdc.sra:SRX43 } \\
87449 \text { [13]; https://identifiers.org/ } \\
\text { ncbi/insdc.sra:SRR7517443 [14]; } \\
\text { https://identifiers.org/ncbi/insdc } \\
\text {.sra:SRR7525090 [15]) }\end{array}$ \\
\hline Illumina NextSeq 500 (new) & UTI89 - NextSeq 500 & FASTQ & $\begin{array}{l}\text { NCBI Sequence Read Archive (https:// } \\
\text { identifiers.org/ncbi/insdc.sra:SRX42 } \\
23297 \text { [16]; https://identifiers.org/ } \\
\text { ncbi/insdc.sra:SRR7349974 [17]) }\end{array}$ \\
\hline $\begin{array}{l}\text { Oxford Nanopore MinlON Mk 1b FLO- } \\
\text { MIN106 (R9.4) (new) }\end{array}$ & UTI89 - MinlON R9.4 & FASTQ & $\begin{array}{l}\text { NCBI Sequence Read Archive (https:// } \\
\text { identifiers.org/ncbi/insdc.sra:SRX43 } \\
87499 \text { [18]; https://identifiers.org/ } \\
\text { ncbi/insdc.sra:SRR7517493 [19]) }\end{array}$ \\
\hline Pacific Biosciences RSII (P6-C4) (new) & UTI89 - PacBio P6-C4 & FASTQ & $\begin{array}{l}\text { NCBI Sequence Read Archive (https:// } \\
\text { identifiers.org/ncbi/insdc.sra:SRX50 } \\
58882 \text { [20]; https://identifiers.org/ } \\
\text { ncbi/insdc.sra:SRX5058883 [21]; } \\
\text { https://identifiers.org/ncbi/insdc } \\
\text { sra:SRR8240630 [22]; https://ident } \\
\text { ifiers.org/ncbi/insdc.sra:SRR8240631 } \\
\text { [23]) }\end{array}$ \\
\hline Roche 454 (previous) [4] & UTI89- 454 & FASTQ & $\begin{array}{l}\text { NCBI Sequence Read Archive (https:// } \\
\text { identifiers.org/ncbi/insdc.sra:SRX00 } \\
0179 \text { [24]; https://identifiers.org/ } \\
\text { ncbi/insdc.sra:SRR000868 [25]; } \\
\text { https://identifiers.org/ncbi/insdc } \\
\text {.sra:SRR000869 [26]; https://identifier } \\
\text { s.org/ncbi/insdc.sra:SRR000870 [27]; } \\
\text { https://identifiers.org/ncbi/insdc } \\
\text {.sra:SRR000871 [28]; https://identifier } \\
\text { s.org/ncbi/insdc.sra:SRR000872 [29]; } \\
\text { https://identifiers.org/ncbi/insdc } \\
\text {.sra:SRR000873 [30]) }\end{array}$ \\
\hline Illumina Hiseq 2000 (previous) [5] & UTI89 - HiSeq 2000 & FASTQ & $\begin{array}{l}\text { NCBI Sequence Read Archive (https:// } \\
\text { identifiers.org/ncbi/insdc.sra:ERX63 } \\
2843 \text { [31]; https://identifiers.org/ } \\
\text { ncbi/insdc.sra:ERX632844 [32]; } \\
\text { https://identifiers.org/ncbi/insdc } \\
\text { sra:ERR687900 [33]; https://identifier } \\
\text { s.org/ncbi/insdc.sra:ERR687901 [34]) }\end{array}$ \\
\hline $\begin{array}{l}\text { Oxford Nanopore MinlON R7 (previous) } \\
\text { [6] }\end{array}$ & UTI89 - MinION R7 & FASTQ & $\begin{array}{l}\text { NCBI Sequence Read Archive (https:// } \\
\text { identifiers.org/ncbi/insdc.sra:ERX98 } \\
7748 \text { [35]; https://identifiers.org/ } \\
\text { ncbi/insdc.sra:ERR908493 [36]) }\end{array}$ \\
\hline
\end{tabular}


3. Not every generation of sequencing machine or library preparation method was used.

\section{Abbreviations}

UPEC: Uropathogenic Escherichia coli; UTI: Urinary tract infection; LB: Lysogeny broth; LMP: Long mate pair; PGM: Personal genome machine.

\section{Acknowledgements}

The authors wish to thank Tyson Clarke and Jonas Korlach of Pacific Biosciences for help with sequencing the PacBlo XL/C2 data set. The authors also wish to thank the Next Generation Sequencing Platform and the GERMS Platform at the Genome Institute of Singapore for technical help and useful discussions related to the generation of these data.

\section{Authors' contributions}

SNF performed the 1D Nanopore sequencing and prepared DNA for the P6-C4 PacBio sequencing, carried out data analysis and collation, and wrote the manuscript. ABI SOLiD library preparation was performed by YCC and FY. Ion Torrent library preparation and sequencing were performed by AJK, YHC, XAR and LTL. PacBio sequencing was performed by JLYC, JK, TC, and SWT. Illumina library preparation was performed my KSM. SLC conceived experiments, analysed data, and wrote the manuscript. Illumina, ABI SOLiD, and lon Torrent sequencing were performed by the Genome Institute of Singapore (GIS) Next Generation Sequencing Platform.

\section{Funding}

This work was supported by the National Research Foundation, Singapore (NRF-RF2010-10), the Singapore Ministry of Health's National Medical Research Council under two Clinician-Scientist Individual Research Grants (NMRC/ CIRG/1357/2013 and NMRC/CIRG/1358/2013) and the Genome Institute of Singapore (GIS)/Agency for Science, Technology, and Research (A*STAR). The funders had no role in the design, collection, analysis, or interpretation of the data. The funders had no role in the writing of the manuscript.

\section{Availability of data and materials}

The data described in this Data note can be freely and openly accessed on Genbank. Please see Table 1 for accession numbers. Specifically, the experiment accessions for the newly presented data are: SRX4387579 [7], SRX4225380 [1 1], SRX4387449 [13], SRX4223297 [16], SRX4387499 [18], SRX5058882 [20], and SRX5058883 [21]. The experiment accession for the previously published data are: SRX000179 [24], ERX632843 [31], ERX632844 [32], and ERX987748 [35].

\section{Ethics approval and consent to participate}

Not applicable.

\section{Consent for publication}

Not applicable.

\section{Competing interests}

The authors declare that they have no competing interests.

\section{Author details}

${ }^{1}$ Genome Institute of Singapore, 60 Biopolis Street, Genome, \#02-01, Singapore 138672, Singapore. ${ }^{2}$ Department of Medicine, Yong Loo Lin School of Medicine, National University of Singapore, 1E Kent Ridge Road, NUHS Tower Block, Level 10, Singapore 119228, Singapore. ${ }^{3}$ Pacific Biosciences, 1305 O'Brien Dr, Menlo Park, CA 94025, USA.

Received: 25 April 2020 Accepted: 14 October 2020

Published online: 20 October 2020

\section{References}

1. Blattner FR, Plunkett G 3rd, Bloch CA, Perna NT, Burland V, Riley M, et al. The complete genome sequence of Escherichia coli K-12. Science. 1997;277:1453-62.

2. Welch RA, Burland V, Plunkett G 3rd, Redford P, Roesch P, Rasko D, et al. Extensive mosaic structure revealed by the complete genome sequence of uropathogenic Escherichia coli. Proc Natl Acad Sci USA. 2002;99:17020-4

3. Hayashi T, Makino K, Ohnishi M, Kurokawa K, Ishii K, Yokoyama K, et al. Complete genome sequence of enterohemorrhagic Escherichia coli O157:H7 and genomic comparison with a laboratory strain K-12. DNA Res. 2001:8:11-22.

4. Chen SL, Hung C-S, Xu J, Reigstad CS, Magrini V, Sabo A, et al. Identification of genes subject to positive selection in uropathogenic strains of Escherichia coli: a comparative genomics approach. Proc Natl Acad Sci USA. 2006:103:5977-82.

5. Sullivan MJ, Ben Zakour NL, Forde BM, Stanton-Cook M, Beatson SA. Contiguity: contig adjacency graph construction and visualisation; 2015. https://doi.org/10.7287/peerj.preprints.1037v1.

6. Sović I, Šikić M, Wilm A, Fenlon SN, Chen S, Nagarajan N. Fast and sensitive mapping of nanopore sequencing reads with GraphMap. Nat Commun. 2016;7:11307.

7. https://identifiers.org/ncbi/insdc.sra:SRX4387579. Accessed 17 May 2020.

8. https://identifiers.org/ncbi/insdc.sra:SRR7517573. Accessed 17 May 2020

9. https://identifiers.org/ncbi/insdc.sra:SRR8247388. Accessed 17 May 2020.

10. Guzman LM, Belin D, Carson MJ, Beckwith J. Tight regulation, modulation, and high-level expression by vectors containing the arabinose PBAD promoter. J Bacteriol. 1995;177:4121-30.

11. https://identifiers.org/ncbi/insdc.sra:SRX4225380. Accessed 17 May 2020.

12. https://identifiers.org/ncbi/insdc.sra:SRR7352157. Accessed 17 May 2020.

13. https://identifiers.org/ncbi/insdc.sra:SRX4387449. Accessed 17 May 2020

14. https://identifiers.org/ncbi/insdc.sra:SRR7517443. Accessed 17 May 2020.

15. https://identifiers.org/ncbi/insdc.sra:SRR7525090. Accessed 17 May 2020.

16. https://identifiers.org/ncbi/insdc.sra:SRX4223297. Accessed 17 May 2020.

17. https://identifiers.org/ncbi/insdc.sra:SRR7349974. Accessed 17 May 2020.

18. https://identifiers.org/ncbi/insdc.sra:SRX4387499. Accessed 17 May 2020.

19. https://identifiers.org/ncbi/insdc.sra:SRR7517493. Accessed 17 May 2020.

20. https://identifiers.org/ncbi/insdc.sra:SRX5058882. Accessed 17 May 2020

21. https://identifiers.org/ncbi/insdc.sra:SRX5058883. Accessed 17 May 2020.

22. https://identifiers.org/ncbi/insdc.sra:SRR8240630. Accessed 17 May 2020.

23. https://identifiers.org/ncbi/insdc.sra:SRR8240631. Accessed 17 May 2020.

24. https://identifiers.org/ncbi/insdc.sra:SRX000179. Accessed 17 May 2020.

25. https://identifiers.org/ncbi/insdc.sra:SRR000868. Accessed 17 May 2020.

26. https://identifiers.org/ncbi/insdc.sra:SRR000869. Accessed 17 May 2020

27. https://identifiers.org/ncbi/insdc.sra:SRR000870. Accessed 17 May 2020.

28. https://identifiers.org/ncbi/insdc.sra:SRR000871. Accessed 17 May 2020

29. https://identifiers.org/ncbi/insdc.sra:SRR000872. Accessed 17 May 2020

30. https://identifiers.org/ncbi/insdc.sra:SRR000873. Accessed 17 May 2020.

31. https://identifiers.org/ncbi/insdc.sra:ERX632843. Accessed 17 May 2020.

32. https://identifiers.org/ncbi/insdc.sra:ERX632844. Accessed 17 May 2020.

33. https://identifiers.org/ncbi/insdc.sra:ERR687900. Accessed 17 May 2020

34. https://identifiers.org/ncbi/insdc.sra:ERR687901. Accessed 17 May 2020

35. https://identifiers.org/ncbi/insdc.sra:ERX987748. Accessed 17 May 2020.

36. https://identifiers.org/ncbi/insdc.sra:ERR908493. Accessed 17 May 2020.

\section{Publisher's Note}

Springer Nature remains neutral with regard to jurisdictional claims in published maps and institutional affiliations. 